DOI: $10.20472 /$ IAC.2018.043.005

\title{
ROSER BONO
}

University of Barcelona , Spain

MARÍA J. BLANCA

Department of Psychobiology and Behavioral Science Methodology, University of Malaga , Spain

\section{RAFAEL ALARCÓN}

Department of Psychobiology and Behavioral Science Methodology, University of Malaga, Spain

\author{
JAUME ARNAU
}

Department of Social Psychology and Quantitative Psychology, University of Barcelona , Spain

\section{THE EFFECTS OF AUTOCORRELATION AND NUMBER OF REPEATED MEASURES ON GLMM ROBUSTNESS WITH ORDINAL DATA}

\begin{abstract}
:
Longitudinal studies involving ordinal responses are widely conducted in many fields of the education, health and social sciences. In these cases, when units are observed over time, the possibility of auto-correlation between observations on the same subject exists. Therefore the assumption of independence which underlines the generalized linear models is violated. Generalized linear mixed models (GLMMs) accommodate repeated measures data for which the usual assumption of independent observations is untenable, and also accommodate a non-normally distributed dependent variable (i.e. multinomial distribution for ordinal data). Thus, GLMMs constitute a good technique for modelling correlated data and ordinal responses. In this study, for a split-plot design with two groups for the between-subjects factor and five response categories, we investigated empirical Type I error rates in GLMMs. To this end, we used a computer program developed by Wicklin to generate longitudinal ordinal data with SAS/IML. We manipulated the total sample size, the coefficient of variation of the group size, the number of repeated measures, and the values of autocorrelation coefficient. For each combination 5,000 replications were performed at a significance level of .05. The GLIMMIX procedure in SAS was used to fit the mixed-effects models for ordinal responses with multinomial distribution and the Kenward-Roger degrees of freedom adjustment for small samples. The results of simulations showed that the test is robust for group effect under all conditions analysed. For time and interaction effects, however, the robustness depends on the number of repeated measures and autocorrelations values. The test tends to be liberal with high autocorrelation, different values of autocorrelation in each group and large number of repeated measures. To sum up, GLMMs are a good analytical option for correlated ordinal outcomes with few repeated measures, low autocorrelation, and the same autocorrelation between groups.

This research was supported by grant PSI2016-78737-P (AEI/FEDER, UE) from the Spanish Ministry of Economy, Industry and Competitiveness.
\end{abstract}

\section{Keywords:}

longitudinal studies, generalized linear mixed models, GLIMMIX, ordinal data, robustness

JEL Classification: $\mathrm{C} 12, \mathrm{C} 15, \mathrm{C} 18$ 